Mirai. Estudios Japoneses

ISSN-e: 2531-145X

http://dx.doi.org/10.5209/mira.67534

\title{
España y Japón ante la robotización: un reto fiscal y económico
}

\author{
M. ${ }^{\mathrm{a}}$ Cristina Bueno Maluenda ${ }^{1}$ (iD
}

Resumen. Partiendo de dos ámbitos geográficos muy distantes en el espacio, España y Japón, no obstante, comparten problemas comunes: su demografía y su esperanza de vida. Dichos elementos combinados con el fenómeno de la robotización y automatización del trabajo hacen que se planteen cuestiones de futuro comunes tales como la de abordar el problema de sostenimiento de los gastos públicos y las pensiones. En este artículo se ofrecen los presupuestos que, desde una perspectiva de política fiscal y económica, revelan la necesidad de planificar posibles soluciones y se repasan las acciones que sendos Gobiernos han comenzado recientemente o están comenzado para abordar este reto, que no exclusivo ni de España ni Japón, pero que sí les resulta si cabe más acuciante por sus propias características sociales. En la última parte, se clasifican las tendencias actuales a propósito de la posibilidad de introducir un impuesto a los robots que permita contribuir a la previsible disminución de ingresos tributarios por causa de la tecnología.

Palabras clave: Robot; automatización; mercados laborales; Japón; robot tax; inteligencia artificial; sistema impositivo; bienestar social; política fiscal.

\section{[en] Spain and Japan in the face of robotization: a fiscal and economic challenge}

Abstract. Starting from two very distant geographical areas in space, Spain and Japan, however, share common problems: their demographics and their life expectancy. These elements, combined with the phenomenon of robotization and work automation, raise common questions for the future, such as addressing the problem of sustaining public expending and pensions. This article provides information that, from a fiscal and economic policy perspective, reveal the need to plan possible solutions and reviews the actions taken in both areas to address this challenge, which is not exclusive to Spain or Japan but is more pressing due their social characteristics. In the last part, current trends are classified regarding the possibility of introducing a tax on robots that contributes to the foreseeable decrease in tax revenues due to technology. Keywords: Robot; automation; labor markets; Japan; Robot-tax; AI artificial intelligence; tax system; social welfare; fiscal policy

Sumario. 1. Introducción: el imparable avance de la robotización. 2. España y Japón: diferentes e iguales frente al reto de la robotización. 3. El impacto de la llamada « $4^{\circ}$ Revolución industrial» en el empleo. 4. Tendencias de política fiscal. ¿Combatir los efectos perversos versus fomentar los efectos beneficiosos? 5. Bibliografía

Cómo citar: Bueno Maluenda, M. C. España y Japón ante la robotización: un reto fiscal y económico, en Mirai. Estudios Japoneses, 4, 2020, 49-59.

\section{Introducción: el imparable avance de la robotización}

Estamos en una era de transformaciones profundas para la humanidad. No se trata (solo) de una nueva revolución tecnológica, la llamada «Cuarta revolución industrial» que también, sino de un cambio de paradigma en las relaciones laborales, tributarias, económicas y sociales.

¿Qué si no se puede pensar del hecho de que haya habido un país que ha otorgado la ciudadanía a un ser cibernético? En este momento que «Sophia», así se llama el robot creado por Hanson Robotics, pueda pasearse por el mundo como una starlet de Hollywood concediendo entrevistas a numeroso público nos parece un hecho ciertamente anecdótico o curioso, en todo caso interesante, pero al mismo tiempo nos provoca algo de inquietud. Les recomiendo que vean la entrevista que se puede encontrar en Youtube $e^{2}$ del famoso actor norteamericano Will Smith a Sophia.

Universidad de Zaragoza (España). Proyecto de referencia "Entornos Laborales Robotizados. Estudio de las Propuestas Japonesas para la Sostenibilidad del Gasto Público y su aplicabilidad en la UE" de la convocatoria 2018 de "Proyectos de I+D Retos Investigación” del Ministerio de Ciencia, Innovación y Universidades. Mail: cribueno@unizar.es.

2 Es un video corto en formato entrevista. El actor trata de provocar reacciones al robot ofreciéndole algo para beber o en el final del corto, aparentando un sentimiento amoroso por ella. Smith, Will (2018). 
Así pues, la «Cuarta Revolución Industrial» no es industrial, ya que afecta a todos los sectores económicos y no solo a la industria pesada. El sector de la sanidad, de los servicios sociales, del turismo, de la exploración espacial, del entretenimiento, el transporte, la protección medioambiental, para combatir la soledad de los ancianos, los servicios de información o comunicación, etc, etc, etc. Y estos son los robots que tienen forma física. Robots que cada vez están más perfeccionados en cuanto a su aspecto físico, que son guapos -guapas-pues abundan las representaciones femeninas humanas ${ }^{3}$.

Si en el aspecto físico y en su desenvolvimiento en nuestro medio urbano los especialistas en robótica están avanzando muy rápido mejorando cada vez más la forma en que caminan, corren o se mueven; aumentando su estabilidad o su equilibrio en circunstancias adversas; mejorando sorprendentemente la gestualidad del ingenio para simular la expresión de sentimientos o reacciones espontáneas en un ser humano ${ }^{4}$; si todo esto digo es digno de ser tenido en consideración como un hecho que ya ha superado su fase inicial, no menos importante es detenerse en la expansión del fenómeno algorítmico -Inteligencia Artificial (AI) - que impregna muchos de los servicios que se ofrecen en la gran tela de araña. La tecnología del big data, los servicios cloud computing, el block chain son solo los ejemplos más representativos del desarrollo de los sistemas algorítmicos, sin olvidar el emergente Internet de las Cosas y de los Servicios (IoT/IoS, Internet of Things/Internet of Services).

El cambio tecnológico es un hecho y en diversos foros internacional se está estudiando cuál va a ser la repercusión en el ámbito laboral. Con independencia del balance -positivo o negativo-que ofrezca la progresiva robotización de la economía y de la sociedad, esto es, si la destrucción del empleo será un grave problema o una cuestión que pueda ser tratable y controlable porque en su lugar puedan aparecer otros nichos laborales, lo que no cabe duda es que el mercado laboral se transformará.

Japón, en el Informe sobre el Futuro del Trabajo dado por el Ministerio de Salud, Trabajo y Bienestar de 2016 prevé que en $2035^{5}$ los modos de trabajo cambiarán en varios sentidos: el trabajo no se verá constreñido por el tiempo ni por el espacio; las formas de trabajo se acompañarán de otros propósitos además del de obtención de lucro en términos de colaboración y responsabilidad sociales; la transformaciones aludidas también contagiarán a las estructuras organizativas de las empresas y sociedades, que necesariamente tendrán que adaptarse rápidamente e introducir elementos de flexibilización; junto a esto, la figura del empleado "full-time" como incentivo para fidelizar al personal de la empresa se desvanecerá y/o transformará en algo diferente, así como los empleados temporales o a tiempo parcial. Ello es debido a que según las previsiones de futuro, los proyectos de trabajo pueden tener una proyección temporal larga pero no así quienes se vinculan a ellos y la permeabilidad de los proyectos entre las empresas será la regla.

Junto a esto, la demanda de trabajadores también sufrirá cambios. Las empresas serán las que realizarán esfuerzos para hacer más atractiva su oferta de trabajo para competir con los trabajadores más adecuados para sus proyectos. Las empresas adoptarán el rol de "project-type organizations". Por ese motivo, los trabajadores podrán estar desarrollando múltiples proyectos simultáneamente, incluyendo también colaboraciones con organizaciones sin ánimo de lucro. Se hará muy necesaria la actualización de las destrezas, de las competencias y de los conocimientos.

Desde el punto de vista de la vida en sociedad, el informe de los expertos japoneses señala que el sentimiento de pertenencia del trabajador a su empresa desaparecerá y la empresas deberán abordar el desafío de funcionar como comunidades simuladas en entornos virtuales apoyados por las $\mathrm{TIC}^{7}$. Ahora bien, por otra parte el hecho de que los trabajadores compartan el mismo campo del saber les hará formar comunidades más fuertes que las que se darían de trabajar en la misma empresa. En este sentido, los sindicatos de trabajadores deberían evolucionar en paralelo.

Una esperanza para el mundo rural. Así se espera en Japón para el año 2035 por la desubicación de los trabajos y la frontera entre los sectores primario a terciario será cada vez más débil. Se ha acuñado el término "Glocalization" 8 para describir el fenómeno por el cual lo local deviene global.

Por otra parte, el Informe intuye que esta transformación del mundo del trabajo supla la falta de personal dedicado a ciertos trabajos, particularmente en los sectores dedicados al cuidado de las personas por razón de su edad - personas de edad o menores- o por su condición física - enfermedad o accidente-.

En Japón, Erica es un robot humanoide que, a juicio de su creador, tiene alma. Forma parte del proyecto: Ishiguro Symbiotic Human-Robot Interaction Project (2020). Erica tiene su propio canal en youtube en el que demuestra sus habilidades como robot-conversador. Se hacen eco de la noticia: Harrison, George / Graham, Ben (2020).

Erica forma parte de los proyectos desarrollados por la ERATO, la Exploratory Research for Advanced Technology, dependiente de la Japan Science and Technology Agency (TSJ) que desde 1981 destina fondos para "promover proyectos de investigación creativos y que representen un desafío científico». Todo ello en el marco de los sectores estratégicos nacionales definidos por la citada Agencia japonesa. Por cierto, el nombre «Erato» era el nombre de la diosa griega de la poesía romántica y también el acrónimo de "Exploratory Research for Advanced Technology". A este tipo de robots se encuadra dentro de los "Symbiotic Human-robot" denominación utilizada por el profesor Ishiguro en su proyecto de investigación.

4 Remito de nuevo a youtube, un canal especializado en la difusión de novedades en materia de robótica. En el siguiente video pueden observar diferentes ejemplos de robots humanoides o formas de animal en diferentes facetas del mundo laboral y social. Enrigue8 (2018).

Ministry of Health, Labour and Welfare (2016): 9-15.

Ministry of Health, Labour and Welfare (2016): 11.

En el entorno internacional a este fenómeno se le está denominando «bitwork» y a los trabajadores «bitworkers», expresiones utilizada por Brown, G. y Whittle, R. (2020): capítulo 4 en el que describen la futura realidad del trabajo y de sus agentes, a los que se les atribuirá las condiciones de flexibilidad, desvinculación de las empresas y multiproyecto ya definidas en el Informe japonés del año 2016.

8 Ministry of Health, Labour and Welfare (2016): 2. 
Japón confía en que la robótica-IA contribuya de manera decisiva a paliar los problemas que en este momento ya se producen en la búsqueda de personas que quieran desempeñar estos cometidos. En este sentido, el sentimiento japonés hacia los robots es radicalmente diferente del que se tiene en Europa. Me parece revelador el titular de una noticia de prensa ${ }^{9}$ que se publicó recientemente que se preguntaba ¿Por qué Japón no tiene miedo a los robots? A juicio del periodista tanto Estados Unidos como la Unión Europea están muy preocupados por la destrucción de empleo por el avance de la automatización de procesos y la irrupción de la robótica en el mundo laboral. Sin embargo en Japón, las encuestas si bien revelan que un amplio porcentaje del $83 \%$ piensa que la automatización supondrá un aumento de las desigualdades, también señalan que los japoneses consideran que la robotización no les hará más duro encontrar trabajo y en un porcentaje muy alto se considera que una economía basada en robots como fuerza de trabajo será mucho más eficiente.

Las razones aducidas, en el citado artículo, para que Japón no tenga miedo a los robots son básicamente las siguientes: Japón tiene uno de los porcentajes de uso de robots más altos del mundo ${ }^{10}$. Otra explicación se encuentra en la cultura popular - películas, series de televisión, novelas, etc.- que han confeccionado un modelo de robot amigable y defensor de la humanidad frente a las versiones aterradoras y apocalípticas americanas y europeas. Y una tercera, la demografía y la incorporación de las mujeres al mundo del trabajo. Falta mano de obra que no es cubierta por empleados extranjeros. Pero la más importante razón es la relativa al apoyo institucional efectivo (funds) del Gobierno a sus ciudadanos para ayudarles a la adaptación. A estas tres, habría que añadir una cuarta, la productividad. En 2017, una compañía de seguros japonesa sustituyó a una treintena de trabajadores por el programa de IBM, Watson Explorer, debido a que la introducción del sistema de inteligencia artificial hacía más rápida la gestión documental de los expedientes sanitarios y de los procesos administrativos ${ }^{11}$.

\section{España y Japón: diferentes e iguales frente al reto de la robotización}

Si en algo España se parece a Japón es en la demografía. Los datos no son nada alentadores si ponemos el foco en su crecimiento vegetativo.

En cuanto a la demografía ${ }^{12}$, está previsto que la población japonesa sea de 112 millones en 2035, unos 15 millones de personas inferior a la actual situada en 127 millones. Al mismo tiempo la población de edad se incrementará debido a la mayor esperanza de vida: la ratio crecerá del actual 26,7\% al 33,4\%. Junto esto, a lo largo de la segunda mitad del siglo XX se ha producido una concentración de la población en las zonas urbanas con la consiguiente despoblación de los núcleos rurales ${ }^{13}$. En España, el fenómeno de la despoblación rural, no siendo exclusivo de España, se distingue del producido en toda Europa, por su aparición súbita y su rápido desarrollo ${ }^{14}$. Todo ello, evidentemente, transformará las necesidades de servicios de salud, de bienestar social y de pensiones.

De acuerdo con los datos del Instituto Nacional de Estadística de España (INE), en el año 2018 la esperanza de vida al nacimiento en España se sitúa en 83,19 años ${ }^{15}$.

En los últimos diez años el número de nacimientos en España no para de decrecer por lo que el crecimiento vegetativo de la población española -diferencia entre nacimientos y defunciones- lleva siendo negativo desde el año 2015, en una progresión creciente. La dispersión de estos datos por Comunidades Autónomas refleja que solo tres comunidades tienen un saldo vegetativo positivo (Madrid, Baleares y Murcia), junto con las dos ciudades autónomas de Ceuta y Melilla.

\footnotetext{
Tett, G. (2019). Noticia que apareció el 12 de junio de 2019.
}

10 El crecimiento de los robots industriales en el mundo fue de un 6 por ciento. Un $74 \%$ de los robots en el mundo se concentran en cinco países, en orden de importancia son: China, Japón, Estados Unidos, la República de Corea y Alemania. La gráfica que resume los porcentajes de inversión en robótica entre regiones, no solo demuestran el apabullante peso de la región Asia/Australia en el número de robots sino su crecimiento exponencial desde 2008 a 2018, comparándolo con los datos de Europa (segundo lugar) y América (tercer puesto). En cuanto a los países: la República de Corea batió un record al incrementar un 10\% su stock de robots industriales. En segunda posición se encuentra Japón seguido de China en tercer lugar. International Federation of Robotics (2019): 14.

Patrinos, H. A. (2020): 4.

12 Ministry of Health, Labour and Welfare (2016): 4.

13 En el trabajo de Vilaroig Moya, R. (2019): 6 y 13 se hace referencia a este fenómeno de acuerdo con los datos publicados por el Ministerio de Salud, Trabajo y Bienestar de Japón, característica a la que, por cierto, también responde la estructura de la población española. Esta realidad, junto con otras comunes a ambos países, como el envejecimiento poblacional, la rigidez del sistema financiero-tributario local así como la necesidad de cumplimiento del principio constitucional de suficiencia financiera local, llevan al autor a presentar fórmulas de financiación de la hacienda local japonesa novedosas, como el Furusato Nozei, basado en la potenciación del producto local y la recompensa por parte de ciudadanos no residentes en la localidad de origen del producto especie de crowdfunding. Sobre el fenómeno de la despoblación en el medio rural español Collantes, F y Pinilla, V. (2020): 2 y 22, afirman que las causas resultan de una combinación de factores tecnológicos, empresariales, territoriales y sociales, una brecha aún no cerrada entre las condiciones de vida rurales y las urbanas. Por este motivo, a nuestro juicio, la evolución de la tecnología en los medios de producción y de las propias actividades económicas antes que un problema puede convertirse en parte de la solución mediante un adecuado fomento y selección de las actividades se pueden promover las condiciones de riqueza para la fijación de la población.

14 Collantes, F y Pinilla, V. (2019): 16. «Sin embargo, durante las cuatro décadas siguientes (a 1950), la rápida transición de España hacia la modernidad económica y social fue testigo de uno de los procesos de despoblación rural más extremos de Europa. En el espacio de una generación, la población rural española se redujo en más de un $25 \%$...». La cursiva es nuestra. En las páginas 47 a 69 se desglosan los datos, las causas y las etapas del fenómeno en estos años en España.

15 Instituto Nacional de Estadística (2019a). 
Las cifras de población en España en $2019^{16}$ son: total 47.100.396, de ellos 5.023.279 extranjeros. Respecto del año anterior la población española ha aumentado en 168.336 personas pero esto es debido a la inmigración y así, el saldo migratorio positivo compensó el saldo vegetativo negativo. Sin embargo, la población activa en España según el INE en $2020^{17}$ es de 22.504 (miles de personas) cifra que no representa ni la mitad de la población total además, hay más personas activas de entre 40 a 70 años que las que hay de entre 16 a 39 años. En estos últimos 20 años, la población activa en España solo ha aumentado unas 5.213,3 (miles de personas). La población activa en el año 2000 de 16 años en adelante suponía 17.290.7 (miles de personas) y el sector más numeroso estaba en la franja de edad de 25 a 29 años.

De acuerdo con la proyección a futuro para el año $2029^{18}$, el INE estima que la población activa en España la cifra se situará en $21.170,8$ (miles de personas), esto es habrá descendido casi un 6\% respecto de las cifras actuales y el sector de población activa más numeroso estará entre los 50 y 54 años. Si esto no fuera preocupante, se añade el hecho del descenso imparable de la población activa para el año 2029 en las franjas de edad de 16 y 19 años y entre los 20 y 24 años, respectivamente 175,3 (miles de personas) y 1.524,8 (miles de personas). Finalmente, en 2029 también el número total de personas activas de entre 40 a 70 años es superior al de aquellas más jóvenes de entre 16 a 39 años, aproximadamente un $35 \%$ más ${ }^{19}$. A esto se ha de añadir que, de nuevo otro año se cierra con una tasa de paro juvenil ${ }^{20}$ alarmante (un $30,51 \%$ en 2019).

En Japón los gobernantes de hoy en día están realizando una apuesta considerable por la robótica para la cobertura de mano de obra que, de otra forma no pueden conseguir: los sectores industriales, pero también los de salud y cuidados de mayores o recepcionistas, atención telefónica 24 horas son las grandes apuestas del país nipón ${ }^{21}$.

¿Qué estrategia plantea el Gobierno español a este respecto? En 2019 el Ministerio de Ciencia, Innovación y Universidades publicó la «Estrategia Española de I+D+I en Inteligencia Artificial» ${ }^{22}$ que supone el desarrollo por parte de España, como estado miembro de la Unión Europea, del llamado «Plan Coordinado sobre la Inteligencia Artificial» que se aprobó finalizando el año 2018 23. Este plan deriva de la Comunicación de la Comisión Europea al Parlamento Europeo, al Consejo Europeo, al Consejo y al Comité Económico y Social y al Comité de las Regiones, Inteligencia artificial para Europa, COM (2018) 237 final, de 25 de abril de 2018 también llamada la "Estrategia Europea sobre la IA"24.

Conscientes de la repercusión en el empleo y en la sociedad en general, desde Europa también se están dirigiendo los esfuerzos de estudio y reflexión hacia el mercado laboral. Así, la Comisión Europea encargó a un grupo de expertos de alto nivel la redacción de un «Informe sobre el Impacto de la Transformación Digital en los mercados laborales de la Unión Europea», que vio la luz en abril de $2019^{25}$.

Simultáneamente a la publicación del citado Informe, la Comisión Europea celebró la conferencia «El futuro del trabajo: hoy, mañana para todos $\gg{ }^{26}$ con representantes de alto nivel: ministros, instituciones y agencias de la UE, de los gobiernos nacionales, interlocutores sociales, de la sociedad civil y del mundo académico. El objetivo era la adopción de medidas para garantizar que las políticas sociales y de empleo de Europa no se vean sobrepasadas por los avances vertiginosos y disruptivos de la tecnología. En noviembre de 2017 los líderes europeos con JeanClaude Juncker a la cabeza habían cristalizado ya tales preocupaciones en veinte principios y derechos esenciales para el mercado laboral y los sistemas de protección social del siglo XXI. Se trata del documento «Pilar Europeo de Derechos Sociales» ${ }^{27}$.

El documento Pilar Europeo de Derechos Sociales ${ }^{28}$ pone el foco en el problema:

«Los mercados laborales y las sociedades evolucionan rápidamente, con nuevas oportunidades y nuevos desafíos derivados de la globalización, la revolución digital, los cambios en las pautas de trabajo y la evolución demográfica y de la sociedad. Los desafíos, como una desigualdad considerable, el desempleo juvenil y de larga duración o la solidaridad intergeneracional, suelen ser similares en todos los Estados miembros, aunque están presentes en distintos grados».

\footnotetext{
Instituto Nacional de Estadística (2020). Datos tomados del estudio del INE sobre cifras de población a 1 de julio de 2019 y estadísticas de Migraciones del primer semestre de 2019

17 Instituto Nacional de Estadística (2019b).

18 Pueden consultarse los datos por años desde el año 1977 a 2029, por franjas de edad y sexo en Instituto Nacional de Estadística (2019c).

19 En cifras redondas, están en el primer grupo unos 8.330 (miles de personas) frente al grupo más maduro, unos 12.784 (miles de personas).

20 Instituto Nacional de Estadística (2019d). La tasa de paro es el cociente entre el número de parados sobre los activos. En 2019 los datos del INE arrojan que la tasa de paro de los menores de 25 años es de un 30,51\%. Se pueden ver de forma sencilla los datos de la encuesta de población activa (EPA) 2019 en relación con las tasas de paro, tasas de empleo y tasas de actividad.

21 Moreno, V. (2019). Noticia de 11 de julio de 2019.

22 Redactada por el Grupo de Trabajo en Inteligencia Artificial (GTIA) dependiente de la Secretaría General de Coordinación de Política Científica del Ministerio de Ciencia, Innovación y Universidades (editor). E-NIPO: 692-10-005-7

23 Comisión Europea (2018b)

24 Comisión Europea (2018a).

Comisión Europea (2019a)

Comisión Europea (2019b)

Dado en Gothenburg el 17 de noviembre de 2017. Comisión Europea (2017).

Comisión Europea (2017): 7, punto 9.
} 
Y más adelante se afirma que: «El progreso económico y social están estrechamente relacionados» y los derechos sociales reconocidos en dicho documento son el eje sobre el que se habrá de construir un «modelo de crecimiento más inclusivo y sostenible, mejorando la competitividad de Europa y haciendo de ella un mejor lugar para invertir, crear puestos de trabajo y fomentar la cohesión social» ${ }^{29}$.

Un año después de la declaración de Gothenburg, la Comisión Europea promovió una serie de iniciativas para hacer realidad los principios y derechos sociales reconocidos en el Pilar ${ }^{30}$ y puso fondos para ello. El Fondo Social Europeo se dotó con más de 100 billones de euros para el período 2021 a 2027. Con la nueva presidencia en las instituciones, consecuencia de las elecciones europeas de 2019, que no cuenta ni un año de rodaje se está a la espera de nuevas iniciativas y de la evolución de las presentes.

El nuevo presupuesto de la UE para 2020 -Acuerdo del Consejo con el Parlamento Europeo de 25 de noviembre de 2019- se orienta fundamentalmente ${ }^{31}$ a la lucha contra el cambio climático; a la reducción del desempleo juvenil que se declara una prioridad política fundamental -a este respecto es destacable, la Iniciativa de Empleo Juvenil (IEJ) dotada con 145 millones de euros- y al desarrollo rural. Asimismo, declara que mantiene su compromiso con el crecimiento y la competitividad de la economía europea. Sin embargo, los principales ámbitos del presupuesto europeo para 2020 en orden decreciente (millones de euros) ${ }^{1}$ son: $59.900 €$ para «crecimiento sostenible», representa el $21 \%$ del presupuesto total; $58.600 €$ para «cohesión económica, social y territorial» y $25.300 €$ para «competitividad para el crecimiento y el empleo». Cierra el presupuesto las siguientes partidas: para «administración» (10.300€); para «una Europa global» (10.300€) y para «instrumentos especiales» $(600 €)$.

El programa Horizonte $2020^{32}$ fue la mayor apuesta de la Unión Europea en materia de Investigación e Innovación y destinó 80 billones de euros en el período 2014 a 2020 que ahora se cierra. Dentro de este programa destacamos la convocatoria de ayudas «FET Innovation Launchpad $\rangle^{33}$ incentiva las iniciativas más punteras en futuras tecnologías. Las ayudas FET (Future and Emerging Technologies) promueven lo que serán las «semillas para un futuro liderazgo industrial y nuevas formas de abordar los desafíos de la sociedad». Entre ellos la bio-robótica o bioingeniería; el sector de las Neuromorphic Computing Technologies (NMC) conjugando estas con las necesidades de la industria en sectores como la automoción, la robótica industrial, análisis big data, aprendizaje y adaptación a contextos operacionales cambiantes; micro-robots IA para la monitorización del entorno y la evolución medioambiental. Una parte específica del programa, además, se destinará al objetivo de la robótica, interfaces e inteligencia artificial para el desarrollo de robots más adecuados a la interacción con los humanos ${ }^{34}$. Aunque el dinero destinado a estas ayudas se ha ido incrementando en términos globales desde 2018 solo representa una pequeña parte del presupuesto total de la $\mathrm{UE}^{35}$.

Japó $^{36}$ por su parte lleva desarrollando estrategias de desarrollo de IA bastantes años antes.

España, por su parte, si bien puede beneficiarse de los programas europeos se encuentra bastante alejada de la media de inversión en $\mathrm{I}+\mathrm{D}$ de los Estados miembros ${ }^{37}$. En la situación actual de prórroga de presupuestos y a la espera de unos nuevos solo cabe imaginar un futuro en el que la cifra supere los índices previos para poder afrontar los compromisos del documento marco la «Estrategia Española de I+D+I en Inteligencia Artificial» en particular, para la definición y concreción de la propia «Estrategia Nacional de Inteligencia Artificial» ${ }^{38}$. Lo positivo en esta cuestión, según se reconoce en el Documento «Estrategia Española...» es que:

«Actualmente, la comunidad académica y científica española dedicada a las tecnologías de IA se caracteriza por su reconocimiento internacional en la mayoría de sus áreas, como son: aprendizaje automático, optimización heurística, planificación, deducción automática, ontologías, lógica y razonamiento, big data, procesamiento del lenguaje natural, visión artificial, robótica, sistemas multiagente, sistemas de recomendación, cooperación hom-

29 Comisión Europea (2017): 7, punto 11.

30 Comisión Europea (2018c). Se trataba de las iniciativas sobre la Autoridad Laboral Europea que la Comisión preveía que empezase a caminar en 2019; sobre la conciliación laboral, llamada iniciativa «Work-life balance for parents and carers», propuesta de Directiva COM(2017) 253 final, esta que decayó el 20-06-2019; la nueva Directiva sobre Transparencia y Predictibilidad de las condiciones de trabajo Directiva 2019/1152/UE, del Parlamento Europeo y del Consejo de 20 de junio de 2019 relativa a unas condiciones laborales transparentes y previsibles en el Unión Europea; de la reforma sobre las reglas de coordinación de la seguridad social que afectan a más países como Islandia, Liechtenstein, Noruega y Suiza, en marzo de 2019 se llegó a un acuerdo provisional (véase la propuesta de 25 de marzo de 2019 en: Council of the European Union (2019)) y una propuesta para que el nuevo presupuesto europeo ayudase a los estados miembros a invertir en las personas.

31 Comisión Europea (2019c)

32 Comisión Europea (2014).

33 Referido al período 2018-2020. Comisión Europea (2018d).

34 Comisión Europea (2018d): 39. Los objetivos específicos son: «Robotics, Interfaces and Artificial Intelligence: a new generation of robotics technologies including soft and flexible robotics, bio-inspired robotics, new approaches to human-machine interaction and cooperation, cognition and artificial intelligence, giving rise to much smarter systems performing sophisticated functions opening radically new opportunities to address societal and economic challenges».

35 De acuerdo con Programa citado, se destinarán 546,15 millones de euros. Comisión Europea (2018d): 62.

36 En 2015, se lanzó la Estrategia y Plan de Acción de Japón sobre Robots en la que se reconoce al país como "superpotencia” en robótica. El nivel de concreción de las acciones a desarrollar es envidiable y debería ser un referente para la implementación de la estrategia española, la cual está como veremos, por abordar.

37 Véase, por ejemplo, sobre sus respectivos PIB, la media europea es de un 2,07\%, en Francia o Alemania, un 3\% en tanto que España solo destina un $1,2 \%$ de su PIB. Frente a ello EEUU dedica un 2,23 de su PIB (que es 20 veces el español). Cinco Días-Tribuna economía (2019). En el mismo sentido: RTVE.es/EFE (2019) y El Economista.es (2019). [Consultados: 28/01/2020].

38 Presidencia de Gobierno (2019). 
bre-máquina, modelado basado en agentes inteligentes, así como por el desarrollo de aplicaciones innovadoras en gran número de sectores estratégicos como la salud, la agricultura, industrias culturales y basadas en la experiencia, servicios y sostenibilidad energética y del medio ambiente ${ }^{39}$.

Sin embargo, a España le queda mucho camino que recorrer y muchas reuniones que realizar para desarrollar los compromisos de esta Estrategia Española y convertirlos en la Estrategia Nacional y sus concretas líneas de acción. Considerando además que hemos empezado con retraso en relación con otros ámbitos, incluido el Europeo.

\section{El impacto de la llamada « $4^{\circ}$ Revolución industrial» en el empleo}

Dos son las grandes líneas en las que se orientan los académicos en relación con el futuro del trabajo tras la irrupción de los robots inteligentes en los sectores productivos.

De una parte, aquellos que entienden que el «desempleo tecnológico», concepto acuñado por el economista Keynes en $1930^{40}$, será la tendencia predominante tanto en forma de «desempleo por sustitución» como desempleo por causa de la desactualización o falta de preparación de profesionales para desempeñar las nuevas formas de negocio: negocios digitales, en general. A nivel mundial, el número de robots industriales instalados en 2018 creció un 6\%.

En Japón ya es evidente la sustitución de empleados humanos por robots, que son fuerza de trabajo común en muchos sectores como el industrial y el de servicios ${ }^{41}$. Por poner solo un ejemplo, la Kawasaki Heavy Industries, Ltd. desarrolla diversas proyectos en robótica. El "du-Aro", es un tipo de robot colaborativo que puede operar al lado del trabajador humano, en el mismo espacio y de la misma forma que lo haría una persona, lo que es un significativo avance que elimina riesgos en la interacción humano-robot y facilita la incorporación de estas máquinas al proceso. Un segundo tipo es el llamado "Successor", un sistema robótico coordinado por control remoto, otro ejemplo de colaboración robot-humano, ya que el trabajador experto se pone a los mandos del robot para ejecutar las mismas tareas que requieren una habilidad y destreza profesional pero realizadas a través o mediante el robot. Un tercer ejemplo, "Kaleido" es un robot humanoide, mismo tamaño y forma que un humano, diseñado para realizar tareas en entornos en donde el ser humano tiene serias dificultades: espacio, en desastres naturales, en el fondo del océano ${ }^{42}$.

De esta forma, en el primer caso el número de trabajadores humanos podría verse reducido por sustitución; en los dos siguientes, tanto puede ocurrir que el robot desplace a humanos como que conviva junto con los que habría sin estos ingenios, generando eso sí mayores posibilidades de éxito u oportunidades de negocio al alcanzar nichos de negocio que sin la colaboración del robot no se obtendrían.

Por ello, otra línea de opinión se decanta por considerar más probable que la incorporación de los robots de cara al futuro abra una ventana de oportunidades para el empleo, nuevos empleos, nuevas formas de trabajar que en un balance final daría como resultado unos mayores índices de empleabilidad.

Hay una tercera tendencia afirma que en el corto plazo habrá destrucción de empleo y falta de profesionales que cubran los nuevos sectores de demanda laboral por falta de capacitación técnica o por obsolescencia de sus competencias (skills) ${ }^{43}$. El Informe McKinsey (2017) estimó que el 15 por ciento del trabajo en el mundo podría estar automatizado en el año 2030. En el entorno americano se piensa que la caída del empleo se concentrará sobre todo en las zonas rurales o que ya están en crisis en este momento ${ }^{44}$.

Nadie puede conocer el futuro pero en algo sí podemos estar de acuerdo y que ha sido expresado por el European Centre for the Development of Vocational Training (CEDEFOP) en su informe resumido «People, Machines, Robots and Skills» al afirmar que el desempleo tecnológico es un tema recurrente pero la falta de empleo en la era digital dependerá de la inteligencia del hombre, no de la artificial ${ }^{45}$.

\section{Tendencias de política fiscal. ¿Combatir los efectos perversos versus fomentar los efectos beneficiosos?}

El reto ahora es para el Derecho, la filosofía ${ }^{46}$ y la ética ${ }^{47}$, pues los tres deben ir de la mano, junto con la formación de nuevas competencias en el sector de la educación, puntal imprescincible para que el desarrollo de tecnología no deje por el camino a sectores de la población.

\footnotetext{
Presidencia de Gobierno (2019): 18

Keynes, J. M. (1931): capítulo 2; (1963): 358-373.

1 Redacción interempresas (2019) e International Federation of Robotics (2019): 13.

42 Toshiya, O. (2019): 5-8. Puede consultarse también la página web de la Japanese Robot Association (JARA) -www.jara.jp- que ofrece información de la industria robótica japonesa, su producción, datos de importación y exportación, etc.

43 Es muy interesante y excelentemente documentado, el Informe del grupo de expertos de alto nivel creado en el seno de la Comisión Europea «El Impacto de la Transformación Digital en los Mercados de Trabajo Europeos» de abril de 2019. Comisión Europea (2019a): 22-28.

44 Rubin, R. (2020)

45 La traducción al español del original en inglés. CEDEFOP (2017a): 1.

46 «The digital is forcing us to rethink new solutions for new forms of agency. While doing so we must keep in mind that the debate is not about robots but about us, who will have to live with them, and about the kind of infosphere and societies we want to create. We need less science fiction and more philosophy». Floridi, L. (2017): 4.

47 Müller, V. (2020).
} 
Dejando de lado las transformaciones en el mundo laboral y en la sociedad en general se abre el debate de cómo financiar la Europa social en este tránsito, porque en algo hay acuerdo, y es que habrá un período de adaptación o transición en el que seremos testigos de la disminución de la ocupación de los trabajadores que se verán sustituidos por robots, particularmente en relación con trabajos repetitivos y automatizables y, al mismo tiempo, a la falta de ocupación de los nuevos trabajos que surjan relacionados directamente con los avances científicos ${ }^{48}$. Estos hombres y mujeres tendrán que estar asistidos por los Estados y sus instituciones.

Es sabido que el gasto público es sostenido principalmente por la recaudación tributaria, los impuestos y el sistema fiscal, en general, cumplen una función instrumental para el desarrollo de los principios constitucionales en todos los Estados de nuestro entorno. No es de extrañar que ante este fenómeno de la robotización y de la digitalización de productos, de negocios, de servicios se plantee el reto de estudiar la viabilidad de fórmulas que, girando en torno al robot o a los sistemas inteligentes, permitan compensar la caída de ingresos tributarios derivados de las rentas de los human-workers.

En el ámbito europeo se han propuesto diversas líneas de desarrollo para afrontar la falta de recursos, bajada de la recaudación en rentas laborales y tributos al consumo en los respectivos impuestos nacionales. Tanto en el seno de las instituciones europeas como la academia se vienen ofreciendo opciones y posibilidades. En un examen conjunto, se podría hablar de tres grandes posiciones al respecto. La vía fiscal innovadora, la vía fiscal conservadora, y la realista. En la primera, si sitúan aquellas propuestas que sugieren la introducción de un impuesto a los robots con planteamientos quizás algo prematuros, como veremos; en la segunda, se encuentran aquellos que opinan de forma más cauta, que es posible orientar las figuras tributarias existentes, consolidadas y adaptarlas al nuevo fenómeno. De acuerdo con ello, el sistema fiscal actual es suficientemente flexible y cuenta con una ventaja adicional, su fundamento constitucional-tributario reconocido por la jurisprudencia. La última, se pregunta hasta qué punto la nueva realidad puede ser objeto de nuevos tributos y en qué momento temporal podrían ser desarrollados.

En Europa, uno de los primeros que abrió la veda de un impuesto innovador, bautizado después como «robottax» fue la Comisión de Asuntos Jurídicos del Parlamento Europeo que se incorporó a su Informe de 2017, con Recomendaciones destinadas a la Comisión Europea sobre normas de Derecho civil sobre robótica. En el apartado $\mathrm{K}$ de este documento se afirmaba que el desarrollo de la robótica y la inteligencia artificial podría dar lugar a que los robots asuman gran parte del trabajo dejando en el aire la viabilidad de los sistemas de seguridad y bienestar sociales, jubilación, reciclaje profesional, desigualdad en el reparto de la riqueza por lo que recomienda la posibilidad de estudiar «someter a impuesto el trabajo ejecutado por robots o exigir un gravamen por el uso y mantenimiento de cada robot, a fin de mantener la cohesión social y la prosperidad ${ }^{49}$.

La mayoría de los miembros del Parlamento europeo (MEP) rechazó el concepto de un «robot-tax». El vicepresidente de la Comisión, ANSIP, también manifestó su opinión contraria por ser contraproducente para los intereses de la Unión Europea pues otros ámbitos geográficos podrían ocupar el lugar de aquella ${ }^{50}$. Tras esta tentativa fueron muchas las manifestaciones contrarias a la idea, sin embargo uno de sus defensores más conocidos era el señor Bill Gates $^{51}$.

La idea de hacer gravar a los robots plantea desde el punto de vista tributario una serie de incertidumbres que se refieren y afectan a los mismos cimientos del Derecho Tributario. En primer término, ¿de qué tipo de robot estamos hablando, de los robots físicos o de los algoritmos? ¿Es un robot un ente susceptible de ser sujeto de una obligación tributaria? ¿Entrarían los robots en el constitucional «todos contribuirán» del artículo 31.1 de la Constitución española? ¿Podríamos aplicar los tributos existentes a estos nuevos sujetos tributarios o tendríamos que crear tributos ad hoc? En tal caso, ¿con qué fundamento desde el punto de vista de la capacidad económica podrían construirse? ¿Habrían de ser tasas, contribuciones especiales o impuestos? Por otra parte, pensando en la inteligencia artificial o en sistemas inteligentes, ¿qué reglas de localización se podrían articular si los conceptos de territorio, frontera, incluso la misma materialidad del sujeto le son tan ajenos como la literatura a un fósil? ¿Debería cambiar la estructura de imposición tradicional basada en propiedades, consumo de bienes y de servicios por otra novedosa como el consumo, uso y la generación de datos?

En la doctrina española el profesor García Novoa ${ }^{52}$ cuestiona la implantación de nuevos impuestos «si no superan un elemental test de racionalidad y que, por el contrario generan una nociva complejidad en el ordenamiento tributario». Ha acuñado el concepto «futurismos fiscales» para designar precisamente la necesidad de reflexionar sobre cuáles han de ser las bases jurídico-tributarias, principalmente, el respeto al principio de capacidad económica que puedan sostener un tributo de esas características. En su opinión, merecería más la pena destinar esfuerzos en la reconsideración y adaptación del sistema fiscal, de las figuras que lo componen para conseguir el efecto deseado. El trabajo de Fernández $\mathrm{Amor}^{53}$ se centra en ese objetivo en la primera parte de su trabajo al analizar las consecuencias fiscales de la entrada de los robots en el sistema productivo versus personas en relación con el principio de neutralidad

\footnotetext{
48 Véanse las previsiones, entre otros por CEDEFOP (2017b). Asimismo, el Dictamen del Comité Económico y social Europeo sobre «El futuro del trabajo. La adquisición de los conocimientos y competencias necesarios para responder a las necesidades de los futuros empleos» (2018/C 237/02), DOUE C 237/8 de 6 de julio de 2018.

49 Parlamento Europeo (2017): 4-5.

50 Véase la noticia en: TH/SW-.04/2017 (2017).

Entre otras, véase la noticia en: Porter, E. (2019).

García Novoa, C. (2018).

53 Fernández Amor, J. A. (2018): 47-96.
} 
fiscal. Se concluye que el robot-worker es fiscalmente más interesante que el human-worker (el robot como activo empresarial es amortizable, por ejemplo, sujeto al principio de prudencia contable y por tanto, a la anticipación de gastos por su reemplazo). Si a esto se suma el hecho de que los robots no tienen costes de cotización es lógico que se plantee la idea de reconsiderar la pertinencia de mantener los beneficios fiscales para I+D +I. Finalmente, se incluye una propuesta de gravamen específico sobre los robots o «robotimpuesto» ${ }^{54}$.

Oberson $^{55}$ anteriormente había expuesto que la sujeción a un impuesto requiere como condición previa ser un sujeto definido y definible al que pueda serle atribuible una capacidad económica separada susceptible de imposición. Ejemplos de entidades sin personalidad jurídica, societarias o no, de conjuntos de bienes y derechos son objeto de tributación en nuestros sistemas fiscales. La cuestión de si un robot podría ser considerado una entidad del artículo 35.4 de la Ley General Tributaria está lejos de ser resuelta por la complejidad del fenómeno «robot». El citado autor Oberson, desde la Universidad de Ginebra plantea la necesidad de conceder «personalidad jurídica a los robots» una personalidad fiscal específica al estilo de las entidades transparentes, requisito previo para el reconocimiento de capacidad económica que poder someter a gravamen. Se formulan dos propuestas o tipos de impuestos: a) un Impuesto por salarios imputados a robots; b) otros ingresos imputables a las actividades de los robots que se gravarían por las cotizaciones sociales; c) creando un Impuesto objetivo (real) a los robots; d) creando un canon o tasa sobre los robots y e) sometimiento de las actividades de los robots al IVA. Dichas propuestas se emiten más como ideas para el debate y articulación posterior que como respuestas cerradas, por ello son muchos los matices y las cuestiones que plantean.

En la línea innovadora también encontramos el trabajo de Abbott y Bogenscheneider ${ }^{56}$ de las Universidades de Surrey (UK) y California (EEUU). que incluye un gravamen a robots denominado «Automation Tax» pero también ponen de relieve una reflexión: la necesidad de revisar las bases sobre las que se fundamentan los sistemas fiscales actuales. En el fondo de esta propuesta está la consideración acerca de que el sistema fiscal incentiva la automatización incluso en los casos en los que la máquina/s no sean más eficientes que la fuerza humana y por ello, ante la necesidad de que los sistemas fiscales sean neutrales para no discriminar a los trabajadores frente a las máquinas sin tener que perder ingresos fiscales. De ahí la razón para el nuevo impuesto ${ }^{57}$.

Es de la misma opinión el Centro Estratégico de Política Europea (EPSC) acerca de la revisión del conjunto del sistema fiscal para reequilibrar el peso de la tributación, alejarlo del trabajo ${ }^{58}$. A este respecto, se señala que el sistema impositivo austríaco es un buen ejemplo de cómo se incentiva a las empresas a invertir en capital humano ${ }^{59}$. Ante la seguridad de que será necesario buscar nuevas vías para incrementar la recaudación, este Centro europeo recoge la idea del «robot tax» que haría contribuir a un robot de forma similar que al trabajador al que desplaza de su puesto de trabajo.

A diferencia de otras propuestas, Europa concede una gran importancia a la inversión privada en $\mathrm{I}+\mathrm{D}+\mathrm{I}$ promovida por el poder público mediante incentivos en los sistemas fiscales. En sus propias palabras: «If public regulations limit innovation, employment is more likely to decrease. An improved tax regime should also be more conducive to supporting AI development in Europe, with all the accompanying economic benefits this entails» ${ }^{60}$.

En marzo de 2018, la Comisión Europea propuso dos iniciativas para asegurar que los negocios digitales fueran objeto de gravamen en la Unión Europea, de una forma justa y que no perjudicase su crecimiento. Una parte significativa de miembros de la UE se opuso al establecimiento, con carácter provisional, de impuesto digital a las empresas por lo que la iniciativa fue abandonada y reemplazada por la propuesta franco-alemana de un tributo temporal de un $3 \%$ sobre las empresas digitales de actividades publicitarias. La propuesta se lanzaba para su entrada en vigor en 2021 por un período de 4 años pero las resistencias en el Consejo hicieron abandonar la idea ${ }^{61}$. Finalmente, se expresaba la necesidad de un acuerdo mundial sobre estas cuestiones, al modo de los Acuerdo Multilaterales desarrollados en el seno de la OCDE.

En la actualidad, la propuestas europeas que están dando pasos más firmes a nivel legislativo son: el Impuesto sobre Servicios Digitales y la propuesta de reformar las reglas de tributación de las empresas que realicen actividades digitales, basadas sobre el concepto de presencia digital significativa (OCDE_UE) a fin de gravar la economía digital y evitar el fraude fiscal ${ }^{62}$.

Fernández Amor, J. A. (2018): 70-78.

55 Oberson, X. (2017): 247-261. Además, pros y contras sobre "robot-tax" también en Walker, J. (2019).

56 Abbott, R./ Bogenscheneider, B. (2018): 145-175. El periódico New York Times se hace eco de las disfunciones del sistema tributario estadounidense sobre la base del estudio de Abbott en la noticia de Porter, E. (2019).

57 En el capítulo III del citado artículo de Abbott y otros (2018): 168 y ss., se desarrollan las opciones que de forma resumida abarcan las siguientes: eliminar las deducciones a las empresas por la incorporación de Automated workers. Esta primera opción es la que según estos autores ha adoptado, parcialmente, Corea del Sur en su "Robot Tax". Creación del un Impuesto sobre la automatización; ofrecer ventajas fiscales para contratar a los trabajadores humanos; incrementar la tributación en el Impuesto sobre Sociedades o bien exigir un Impuesto a las empresas por auto-empleo (Corporate Self-employment Tax). Véase también las propuestas del monográfico sobre la Imposición de la Economía Digital de Haslehner. W, Kofler, G., Pantazatou, K., Rust. A. (2019).

58 Comisión Europea, Centro Estratégico de Política Europea (EPSC) (2019): 105. A su juicio: «The changing nature of word challenges labour taxation and reinforces the need for a tax shift away from labour»).

59 Comisión Europea, Centro Estratégico de Política Europea (EPSC) (2019): 104.

${ }^{60}$ Comisión Europea, Centro Estratégico de Política Europea (EPSC) (2019): 107. Se pone como ejemplo al gobierno de Canadá y sus leyes fiscales que ofrecen generosos incentivos (credit tax) que las sitúan en top 5 mundial según datos de la OCDE.

61 Traducción libre. Comisión Europea, Centro Estratégico de Política Europea (EPSC) (2019): 106.

62 Propuesta de Directiva del Consejo por la que se establecen normas relativas a la fiscalidad de las empresas con una presencia digital significativa, COM (2018) 147 final, de 21 de marzo de 2018 y Propuesta de Directiva del Consejo, relativa al sistema común del Impuesto sobre los servicios digitales que grava los ingresos procedentes de la prestación de determinados servicios digitales, COM (2018) 148 Final, de la misma fecha. 
En otros ámbitos, como el estadounidense, se detectan problemas similares. El Informe MacKinsey de 2019 «The Future of Work in America. People and places, today and tomorrow» ${ }^{63}$, plantea problemas y soluciones similares a las europeas, con matices. En América, el candidato en 2020 a la presidencia de los EEUU (Bill De Blasio) se compromete a introducir un impuesto a las empresas que incorporen a robots que desplacen a los humanos y creará una agencia federal de seguimiento y control de la automatización (FAWPA, Federal Automation and Worker Protection Agency). Al mismo tiempo que revisará el efecto pernicioso que producen los beneficios fiscales de investigación y desarrollo de las empresas que deducen impuestos y cuyo efecto final es la destrucción de empleo. El "robot tax" americano gravaría las grandes Empresas y sería un impuesto finalista cuyos recursos se destinarían a recuperar la fuerza laboral humana desplazada ${ }^{64}$.

Finalmente en Japón ${ }^{65}$, la preocupación no es tanto hacer tributar a los robots como adecuar su sistema de seguridad social y unificar el sistema fiscal a los nuevas formas de trabajo. Estas formas de trabajo que rompen toda barrera de tiempo, espacio edad y sexo ${ }^{66}$ en donde la relación con la empresa, tanto en el lugar de trabajo como en el tiempo de trabajo en cada una de ellas se espera que sean mucho más flexibles y abiertas no se ajustan a los parámetros de la tributación y del régimen de seguridad social actualmente vigente.

En el sistema fiscal japonés ${ }^{67}$, basado en las familias antes que en el individuo, las nuevas formas de trabajo más flexibles desde el punto de vista de su relación con la empresa y la incorporación de las mujeres al mundo laboral hacen que se reconsidere la transformación del sistema fiscal y de seguridad social ${ }^{68}$.

Se considera también necesario que estos cambios no se produzcan en detrimento de otras formas o fuentes de tributación. Es decir, el respeto al principio de neutralidad debe ser efectivo por lo que no podrá discriminar por razones del lugar de trabajo o del tiempo de trabajo. El número de personas que pueden trabajar con satisfacción dependerá de sus ingresos fiscales y de los gastos financieros que se destinen a la realización de estas políticas.

En conclusión, las vías fiscales para abordar e intentar dar una solución al problema de la transformación del mundo del trabajo y de los trabajadores desplazados distan mucho de estar definidas. Como señalaba al principio, estamos inmersos en la transformación, en un vórtice de ideas y de fenómenos novedosos. Corresponde, en este momento, reflexionar y definir los principios sólidos sobre los que asentar los nuevos fundamentos de las relaciones laborales, derechos laborales e imposición de la riqueza y de las nuevas capacidades económicas para, de este modo, poder sustentar las nuevas figuras tributarias, si es que deben existir.

\section{Bibliografía}

Abbott, Ryan / Bogenscheneider, Bret (2018): "Should Robots Pay Taxes? Tax Policy in the Age of Automation". En: Harvard Law and Policy Review, Vol. 12, pp. 145-175. http://harvardlpr.com/wp-content/uploads/2018/03/AbbottBogenschneider.pdf

Barberán Pelegrín, Francisco / Kuroda, Kiyohiko / Okabe, Fuminobu. (coord.) (2013): Introducción al Derecho Japonés Actual. Cizur Menor: Aranzadi. Brown, Gavin y Whittle, Richard (2020): Algorithms, Blockchain and Cryptocurrency: Implications for the Future of the Workplace. Bingley (UK): Emerald Group Publishing.

CEDEFOP (2017a): People, Machines, Robots and Skills, UE. https://www.cedefop.europa.eu/files/9121_en.pdf [Consulta: 20/01/2020].

CEDEFOP (2017b): Briefing Note, julio 2017, People, Machines, Robots and Skills. UE. https://www.cedefop.europa.eu/en/publications-and-resources/ publications/9121. / http://www.cedefop.europa.eu/en/events-and-projects/projects/digitalisation-and-future-work [Consulta: 28/01/2020].

Cinco Días-Tribuna economía (2019): España sigue a la cola de Europa en I+D, Periódico El País, S.L. https:/cincodias.elpais.com/cincodias/2019/09/12/economia/1568301249_285136.html [Consulta: 28/01/2020].

Collantes, Fernando y Pinilla, Vicente (2019): ¿Lugares que no importan? La despoblación de la España rural desde 1900 hasta el presente, Zaragoza, Prensas de la Universidad de Zaragoza.

Collantes, Fernando y Pinilla, Vicente (2020): "La verdadera historia de la despoblación de la España rural y cómo puede ayudarnos a mejorar nuestras políticas". En: Asociación Española de Historia Económica (AEHE), documentos de trabajo, núm. 2001, Madrid, pp. 1-25.

Comisión Europea (2019b): High-Level Expert Group on the Impact of the Digital Transformation on EU Labour Market. UE. https://ec.europa.eu/ commission/presscorner/detail/es/IP_19_2016 [Consulta: 28/01/2020].

Comisión de Asuntos Jurídicos. Parlamento Europeo (2017): "Recomendaciones destinadas a la Comisión Europea sobre normas de Derecho civil sobre robótica". Número A8-0005/2017, de 27 de enero.

Comisión Europea (2014): Horizon 2020. UE. https://ec.europa.eu/programmes/horizon2020/en/what-horizon-2020 [Consulta: 28/01/2020].

Comisión Europea (2017): European Pillar of Social Rights. UE. https://ec.europa.eu/commission/priorities/deeper-and-fairer-economic-and-monetary-union/european-pillar-social-rights_en [Consulta: 28/01/2020].

Comisión Europea (2018a): Comunicación de la Comisión al Parlamento Europeo, al Consejo Europeo, al Consejo, al Comité Económico y Social Europeo y al Comité de las Regiones. Inteligencia artificial para Europa. UE. https:/ec.europa.eu/transparency/regdoc/rep/1/2018/ES/COM-2018237-F1-ES-MAIN-PART-1.PDF [Consulta: 28/01/2020].

63 Por ejemplo, sobre la necesidad de incentivos fiscales o de sistemas de "crédito fiscal" para desempleados tecnológicos durante los periodos de adaptación en McKinsey Global Institute (2019a): 18, 19 y 95. Interesante también su informe sobre la mujer en la era de la automatizacion, McKinsey Global Institute (2019b).

${ }_{64}$ De Blasio, B. (2019). Sobre Estados Unidos puede verse también el anexo del documento Comisión Europea, Centro Estratégico de Política Europea (EPSC) (2019): 146.

65 Ministry of Health, Labour and Welfare (2016): 28.

66 Traducción libre. Ministry of Health, Labour and Welfare (2016): 29.

67 Sobre las características del sistema tributario japonés entre otros: Ishi, H. (2004);. Nishiyama, Y. / Vilarroig Moya, R. (2013): 172-202. Sobre conceptos generales del Derecho japonés, vid: Oda, H. (2011). Barberán, F./ Kuroda, K / Okabe, F. (coord.) (2013).

${ }_{68}$ El debate sobre la financiación y la sostenibilidad de las cuentas públicas en Japón es un tema abierto y en debate, puede verse el reciente trabajo de Kudo, T. (2020): 183-201 y análisis anteriores, desde el punto de vista económico en Doi, T. / Ihori, T. / Mitsui, K. (2006): 1-43. 
Comisión Europea (2018b): Comunicación de la Comisión al Parlamento Europeo, al Consejo Europeo, al Consejo, al Comité Económico y Social Europeo y al Comité de las Regiones. Plan Coordinado sobre la Inteligencia Artificial. COM(2018) 795 final, dado en Bruselas, 7.12 .2018 UE. https://ec.europa.eu/transparency/regdoc/rep/1/2018/ES/COM-2018-795-F1-ES-MAIN-PART-1.PDF [Consulta: 28/01/2020].

Comisión Europea (2018c): Noticias de Prensa. UE. https://ec.europa.eu/commission/presscorner/detail/en/STATEMENT 18 6390 [Consulta: 28/01/2020].

Comisión Europea (2018d): Horizon 2020. Work Programme 2018-2020. UE. https://ec.europa.eu/research/participants/data/ref/h2020/wp/2018-2020/ main/h2020-wp1820-fet_en.pdf

Comisión Europea (2019a): Hight Level Expert Group on the Impact of the Digital Transformation on EU Labour Markets. Final Report. UE. https:// ec.europa.eu/digital-single-market/en/high-level-expert-group-impact-digital-transformation-eu-labour-markets [Consulta: 28/01/2020].

Comisión Europea (2019c): Noticias de Prensa. UE. https://www.consilium.europa.eu/es/press/press-releases/2019/11/18/2020-eu-budget-council-andparliament-reach-agreement/pdf [Consulta 28 de enero de 2020].

Comisión Europea, Centro Estratégico de Política Europea (EPSC) (2019): AI. The future of Work? Work of the Future. On how artificial intelligence, robotics and automation are transforming jobs and the economy in Europe. UE. https://ec.europa.eu/epsc/sites/epsc/files/ai-report online-version. pdf [Consulta: 28/01/2020].

Comité Económico y Social Europeo (2018): "Dictamen sobre «El futuro del trabajo. La adquisición de los conocimientos y competencias necesarios para responder a las necesidades de los futuros empleos" (2018/C 237/02), DOUE C 237/8 de 6 de julio de 2018.

Council of the European Union (2019): Interinstitutional File: 2016/0397. https://data.consilium.europa.eu/doc/document/ST-7698-2019-ADD-1REV-1/en/pdf [Consulta: 28/01/2020].

De Blasio, B. (2019): Why American Workers Need to be Protected from Automation. WIRED. https://www.wired.com/story/why-american-workersneed-to-be-protected-from-automation/?mod=article_inline [Consulta: 28/01/2020].

Doi, Takero / Ihori, Toshihiro / Mitsui, Kiyoshi (2006): "Sustainability, Debt Management, and Public Debt Policy in Japan”. En: NBER Working Papers Series, Cambridge, pp. 1-43.

El Economista.es (2019): La I+D española vuelve al nivel precrisis, pero sigue lejos de Europa. Ecoprensa, S.A. https://www.eleconomista.es/especialtecnologia-startups/noticias/10259591/12/19/La-ID-espanola-vuelve-al-nivel-precrisis-pero-sigue-lejos-de-Europa.html entre otras muchas referencias. [Consulta: 28/01/2020].

Enrigue8 (2018): Top 10 Most Amazing Advanced Robots That Will Change Your World: Youtube. Youtube. https://www.youtube.com/ watch? $=\mathrm{u} 3 \mathrm{vdgJVyKeg}$ [Consulta: 23-01-2020]

Fernández Amor, José Antonio (2018): "Derecho tributario y cuarta revolución industrial: análisis jurídico sobre aspectos fiscales de la robótica". En Nueva Fiscalidad, núm. 1, Madrid, pp-47-96.

Floridi, Luciano (2017): "Robots, Jobs, Taxes, and Responsibilities". En: Philosophy and Technology, 30, pp. 1-4. Published online: 14 March 2017. $<$ doi:10.1007/s13347-017-0257-3>

García Novoa, César (2018): La tributación de los robots y el futurismo fiscal [página WEB], Taxlandia. Blog Fiscal y de Opinión Tributaria. https:// www.politicafiscal.es/cesar-garcia-novoa/la-tributacion-de-los-robots-y-el-futurismo-fiscal [Consulta: 28/01/2020].

Harrison, George / Graham, Ben (2020): Soul Mate. Erica the Japanese robot. The Sun. https://www.thesun.co.uk/tech/5050946/erica-robot-lifelikesoul-jokes [Consulta: 23-01-2020]

Haslehner, Weiner, Kofler, Georg, Pantazatou, Katerina, Rust, Alexander (2019) Tax and the Digital Economy. Challenges and Proposal for Reform. Alphen aan den Rijn: Wolters Kluwer International, Series on International Taxation, núm. 69.

Instituto Nacional de Estadística (2019a): Nota de prensa: Movimiento Natural de la Población/Indicadores Demográficos Básicos. Primer semestre 2019. INE. https://www.ine.es/dyngs/INEbase/es/operacion.htm?c=Estadistica C\&cid=1254736177003\&menu=ultiDatos\&idp=1254735573002 [Consulta: 23-01-2020]

Instituto Nacional de Estadística (2019b): INE.Base: resultados nacionales población activa. INE. https://www.ine.es/jaxi/Datos.htm?path=/t22/ p212/2016-2029/10/\&file=01003.px [Consulta: 23-01-2020]

Instituto Nacional de Estadística (2019c): INE.Base: resultados nacionales población activa. INE. https://www.ine.es/jaxi/Tabla.htm?path=/t22/ p212/2016-2029/10/\&file=01003.px \&L=0 [Consulta: 23-01-2020]

Instituto Nacional de Estadística (2019d): Mercado laboral: Explica. INE. https://www.ine.es/infografias/tasasepa/desktop/index.html?lang=es [Consulta: 23-01-2020]

Instituto Nacional de Estadística (2020): Nota de prensa: Cifras de Población (CP) a 1 de julio de 2019. INE. https://www.ine.es/prensa/cp_j2019_p. pdf [Consulta: 23-01-2020]

International Federation of Robotics (2019): Informe IFR. IFR. https://ifr.org/downloads/press2018/Executive\%20Summary\%20WR\%202019\%20Industrial\%20Robots.pdf [Consulta: 23-01-2020]

Ishi, Hiromitsu (2001): The Japanese Tax System. New York: Oxford University Press, edición 3.

Ishiguro, Symbiotic Human-Robot Interaction Project (2020): Robot. Japan Science and Technology Agency. https://www.jst.go.jp/erato/ishiguro/en/ robot.html [Consulta: 23-01-2020]

Keynes, John Maynard (1931): Essays in Persuasion. Gutenberg Canada. https://gutenberg.ca/ebooks/keynes-essaysinpersuasion/keynes-essaysinpersuasion-00-h.html). [Consulta: 28/01/2020].

Keynes, John Maynard (1963): Essays in Persuasion. New York: W.W. Norton and Co.

Kudo, Takeshi (2020): "Fiscal Consolidation and Sustainability of Japan’s Public Debt after the Global Financial Crisis". En: Keiei to Keizai, vol 99, núm. 1, 2, 3, 4 february, Nagasaki, pp. 183-201.

McKinsey Global Institute (2019a): The Future of Work in America. People and places, today and tomorrow. McKinsey and Company. https://www. mckinsey.com/ /media/McKinsey/Featured\%20Insights/Future $\% 20$ of $\% 20$ Organizations/The $\% 20$ future $\% 20$ of $\% 20$ work $\% 20$ in $\% 20$ America $\% 20$ People\%20and\%20places\%20today\%20and\%20tomorrow/MGI-The-Future-of-Work-in-America-Report-July-2019.ashx [Consulta: 28/01/2020]

McKinsey Global Institute (2019b): The Future of Women at work: Transitions in the age of automation. McKinsey and Company. https://www.mckinsey.com/featured-insights/gender-equality/the-future-of-women-at-work-transitions-in-the-age-of-automation [Consulta: 23-01-2020]

Ministry of Health, Labour and Welfare (2016): "Future of Work: 2035". For Everyone to Shine Report. MHLW. https://www.mhlw.go.jp/file/06Seisakujouhou-12600000-Seisakutoukatsukan/0000152704.pdf [Consulta: 23-01-2020]

Moreno, V. (2019): Los robots y la inteligencia artificial impulsan sus propias leyes. Diario Expansión. www.expansion.com/juridico/actualidad-tende ncias/2019/07/11/5d277679e5fdeae67b8b46bb.html [Consulta: 28 enero 2020].

Müller, Vincent C. (2020): Standford Encyclopedia of Philosophy. Standford University. https://plato.stanford.edu/entries/ethics-ai/ [Consulta: $15 / 05 / 2020]$

Nishiyama, Y. / Vilarroig Moya, R. (2013): “Derecho Tributario”. En Barberán, F./ Kuroda, K / Okabe, F. (coord.): Introducción al Derecho Japonés Actual. Cizur Menor: Aranzadi, pp. 172-202.

Oberson, Xavier (2017): "Taxing Robots? From the Emergence of an Electronic Ability to Pay a Tax on Robots or the use of Robots". En World Tax Journal: WTJ, Vol. 9, núm. 2, mayo 2017, pp-247-261.

Oda, Hiroshi (2011): Japanese Law. New York: OUP Oxford, Edición: 3.

Parlamento Europeo (2017): Informe de la Comisión de Asuntos Jurídicos con Recomendaciones destinadas a la Comisión sobre normas de Derecho civil sobre robótica, de 27 de enero. Número A8-0005/2017. Procedimiento: (2015/2103(INL).

Patrinos, Harry Anthony (2020): “The Learning Challenge in the 21st Century”. En Policy Research Working Paper, 9214, pp. 1-13. 
Porter, Eduardo (2019): Don't Fight the Robots. Tax Them. The New York Times. https://www.nytimes.com/2019/02/23/sunday-review/tax-artificialintelligence.html [Consulta: 28/01/2020].

Presidencia de Gobierno (2019): El Gobierno presenta su proyecto de Estrategia Española para la Inteligencia Artificial en I+D+I. La Moncloa. https://www.lamoncloa.gob.es/presidente/actividades/paginas/2019/040319-sanchez.aspx / https:/www.lamoncloa.gob.es/presidente/actividades/ Documents/2019/040319-estrategia-inteligencia.pdf [Consulta: 28/01/2020]

Redacción interempresas (2019): Crece un 6\% el número de robots industriales instalados en todo el mundo en 2018. Interempresas. https://www.interempresas.net/Robotica/Articulos/254914-Crece-un-6-por-ciento-el-numero-de-robots-industriales-instalados-en-todo-el-mundo-en-2018.html [Consulta: 28/01/2020].

RTVE.es/EFE (2019): En España se gasta la mitad en I+D que en la Unión Europea [página WEB], RTVE. http://www.rtve.es/noticias/20190110/ espana-se-gasta-mitad-id-union-europea/1866683.shtml [Consulta: 28/01/2020].

Rubin, Richard (2020): The "Robot Tax" Debate Heats Up. The Wall Street Journal. https:/www.wsj.com/articles/the-robot-tax-debate-heatsup-11578495608 [Consulta: 28/01/2020].

Smith, Will (2018): Will Smith Tries Online Dating. Youtube. https:/www.youtube.com/watch?v=M19v3wHLuWI

Tett, Gillian (2019): Why Japan isn't afraid of robots?. Financial Times Magazine. https://www.ft.com/content/87ac09b0-8c9a-11e9-a24d-b42f641eca37 [Consulta: 23-01-2020].

TH/SW-.04/2017 (2017): Unconditional basic income and robot tax. Deutsche Sozialversicherung Europavertretung. https:/dsv-europa.de/en/ news/2017/04/robotersteuer.html [Consulta: 28/01/2020].

Toshiya, Okuma, (2019): “Editorial, World Robotics (2019) edition”. International Federation of Robotics (IFR). https://ifr.org/free-downloads/ [Consulta: 28/01/2020].

Vilaroig Moya, Ramón (2019), "Los impuestos de la ciudad natal en Japón: un sistema de financiación local basado en métodos de crowdfunding”. En Documentos de Trabajo del Instituto de Estudios Fiscales, núm 12, Madrid, pp. 1-50.

Walker, John (2019): Robot Tax-A Summary of Arguments "For" and "Against”. EMERJ, The AI Research Company. https://emerj.com/ai-sectoroverviews/robot-tax-summary-arguments [Consulta: 28/01/2020]. 